Review Article

\title{
Celiac disease and its histopathology
}

\section{Pudasaini $\mathrm{S}^{1}$}

\author{
${ }^{I}$ Department of Pathology, Nepal Medical College Teaching Hospital, Jorpati, Nepal.
}

\section{Keywords: \\ Celiac disease; Duodenal biopsy; Enteropathy; Gluten}

\begin{abstract}
Celiac disease is gluten induced enteropathy and is a chronic inflammatory disorder of the small intestine characterized by malabsorption. It is a common immune mediated disorder which is triggered by consumption of wheat (gluten). It occurs in genetically predisposed individuals (carriers of HLA-DQ2 and DQ8 haplotypes). It is characterized by inflammation of the small-intestinal mucosa and myriad gastrointestinal and systemic manifestations. A duodenal biopsy with positive serology is the gold standard for the diagnosis of Celiac disease. As there are changing presentation for Celiac disease, communication of pathologist and gastroenterologists is essential for appropriate interpretation of duodenal biopsy.
\end{abstract}

\section{INTRODUCTION}

Celiac disease $(\mathrm{CD})$ is a common disease, affecting $1 \%$ of the population and evidence suggests that prevalence is increasing. ${ }^{1} \mathrm{CD}$ is known as gluten-sensitive enteropathy, celiac sprue or nontropical sprue. It is a chronic inflammatory disorder of the small intestine characterised by malabsorption after ingestion of wheat gluten or related derivatives of barley and rye in individuals with a certain background. The pathogenesis involves a $\mathrm{T}$ cell mediated immune response and autoreactive B lymphocytes that produce autoantibodies directed against gliadin,

\section{Correspondence:}

Dr. Sujata Pudasaini, MBBS, MD

Associate Professor, Department of Pathology,

Nepal Medical College Teaching Hospital, Jorpati, Kathmandu, Nepal

E-mail: sujatapudasaini@gmail.com endomysium or tissue transglutaminase in individuals with a genetic susceptibility related to HLA- DQ2 and HLADQ8. ${ }^{2,3}$

The clinical manifestations of $\mathrm{CD}$ are changeable in nature and vary markedly with the age of the patient, the duration and extent of disease and the presence of extra intestinal pathological conditions. In addition to classical gastrointestinal form, a variety of other clinical manifestations of the disease has been described including atypical and asymptomatic forms. ${ }^{4}$

Diagnosis of CD is extremely challenging. Serological tests developed in the last two decades provide a non invasive tool to screen both individuals at risk for the disease and the general population. The diagnosis is based on the 
Table 1 : The modified Marsh classification of gluten induced small intestinal damage

\begin{tabular}{cl}
\hline Stage 0 & $\begin{array}{l}\text { Preinfiltrative mucosa, up to } 30 \% \text { of patients with dermatitis herpetiformis (DH) or gluten ataxia have small-intestinal } \\
\text { biopsy specimens that appear normal (fig.1) }\end{array}$ \\
\hline Stage 1 & $\begin{array}{l}\text { Increase in the number of intraepithelial lymphocytes (IELs) to more than } 30 \text { per } 100 \text { enterocytes (fig. 2) } \\
\text { Stage 2 }\end{array}$ \\
$\begin{array}{l}\text { Crypt hyperplasia. In addition to the increased IELs, there is an increase in crypt depth without a reduction in villus } \\
\text { height. Gluten challenge can induce these changes, which can also be seen in } 20 \% \text { of untreated patients with dermatitis } \\
\text { herpetiformis and celiac disease }\end{array}$ \\
$\begin{array}{l}\text { Villous atrophy: A- partial (fig. 3), B- subtotal, C- total (fig.4). This is the classic celiac lesion and is found in } 40 \% \text { of } \\
\text { DH patients. Despite marked mucosal changes, many individuals are asymptomatic and therefore classified as having } \\
\text { subclinical or silent cases. This lesion is characteristic of, but not diagnostic of, celiac disease and can also be seen with } \\
\text { severe giardiasis, infantile food sensitivities, graft versus-host disease, chronic ischemia of the small intestine, tropical } \\
\text { sprue, immunoglobulin deficiencies, and other immune deficiencies and allograft rejection }\end{array}$
\end{tabular}

(Ref: Celiac disease, World Gastroenterology Organization Global Guidelines, April 2012)

detection of specific auto antibodies (anti- transglutaminase type $2 \operatorname{IgA}$ ) and compatible findings at duodenal histology like surface enterocyte damage, increased intraepithelial lymphocytes (IELs), crypt hyperplasia and villous atrophy being considered the principal hallmark. ${ }^{2,4,5}$

The disease should be detected as early as possible because untreated $\mathrm{CD}$ is associated with many severe complications such as intestinal lymphoma, cancer and osteoporosis. ${ }^{4}$

\section{HISTORY}

Celiac disease came into accounts as early as 1st century $\mathrm{AD}$ when the physician Celsus introduced the Latin term "celiac" to indicate a diarrhea like disease. Later in $250 \mathrm{AD}$ and the clinical signs were described by Areteo Cappadocia. However it was Gee, who in 1888 introduced the clinical findings associated with $\mathrm{CD}$ in both adults and children.3,6 Gee was unable to derive an explanation for its pathogenesis and the gross morphology of the small bowel. Also the small bowel biopsy was restricted to autopsy investigations and thus the early attempts to study these tissues were hampered by autolysis. ${ }^{3}$

Paulley et al provided the first histopathological correlation with Celiac disease. Other important contributions were Beneke (1910), Justi (1913) and Manson-Bahr, who recognized the presence of inflammation and villous atrophy in the small intestine with Celiac disease. ${ }^{3}$

\section{PATHOGENESIS}

Celiac disease is widely regarded as an autoimmune disease that arises from an aberrant immune response towards derivatives of gluten, which is present in wheat, barley and rye, in genetically susceptible people. . $^{3,78}$ Other cereals such as rice and millet are considered to be safer, as their proteins bear even less similarity to those of wheat, rye and barley. ${ }^{3}$

Patients with CD have a predominance of HLA Class II DQ2 and/or DQ8 molecules. ${ }^{9}$ An individual not carrying DQ2 and DQ8 alleles is extremely unlikely to develop CD.10 Gliadin derived peptides are processed by HLA Class II molecules for presentation to helper $\mathrm{T}$ cells in susceptible mucosa that has perhaps been primed by a triggering effect, Helper $\mathrm{T}$ cells are activated and there is invasion of the surface epithelial cell by CD8 T cells. It is also proposed that there is direct gliadin toxicity on enterocytes stimulating the HLA molecules. Transglutaminase, (normal gut enzyme) which is released during injury link with gliadin forms a neopeptide. This becomes the target of an antibody response. Thus CD represents a complex array of cellular and humoral immune response. ${ }^{9}$

Transglutaminase has recently been identified as the epitope recognized by the antiendomysial antibody, a sensitive and specific marker of Celiac disease. Elimination of gliadin stops the direct mucosal injury and eliminates the substrate necessary to form the neopeptide that propagates immunologically mediated damage. ${ }^{9,10} \mathrm{~A}$ diverse population of immune mediators contribute to $\mathrm{CD}$ including macrophages, plasma cells, CD4+T helper cells, CD8+ cytotoxic T cells and natural killer cells. ${ }^{3}$ The inflammatory cascade produces inflammatory cytokine, proteinases and other tissue damaging mediators which damages the mucosa leading to characteristic histopathological findings. ${ }^{10}$

\section{CLINICAL SPECTRUM}

Celiac disease occurs both in adults and children with a female predominance (female to male ratio, 2-3:1). ${ }^{10,11}$ The prevalence ranges from $10 \%$ to $13 \%$ in first degree relatives and a high rate of concordance (70 to $75 \%$ ) in monozygotic twins compared to dizygotic twins. ${ }^{3,6,10,12}$ The incidence is higher in wheat eating populations such as Western Europe and North America while the incidence continues to rise in Eastern societies, possibly as a result of western style eating habits. ${ }^{2}$

Clinical presentation varies from full blown malabsorption with weight loss, diarrhea and steatorrhea to more subtle symptoms such as folate or iron deficiency anemia, flatulence, episodic diarrhea, loose stools, neurological problems, osteoporosis and vitamin $\mathrm{K}$ and $\mathrm{D}$ deficiencies in as many as $50 \%$ of patients. Delayed puberty, infertility, protein deficiencies and elevated liver enzyme levels are also seen., ${ }^{2,10,13}$ Celiac disease is also found to be associated with diabetes mellitus type 1 . They also have increased risk 


\section{Table 2: Factors for reliable histological diagnosis \\ Number of biopsy procured \\ Quality of biopsy samples \\ Handling of samples \\ Patchiness of mucosal damage \\ Different grades of lesion \\ Subjective histologic interpretation}

(Ref: Celiac disease, World Gastroenterology Organization Global Guidelines, April 2012)

of sepsis and the risk is higher for pneumococcal related sepsis. ${ }^{11}$ One study done in Italy has shown that children born in summer are at higher risk to develop CD than children born in other seasons. ${ }^{14}$ In children within few months of introducing the child to wheat based foods, the classic syndrome of chronic diarrhea, abdominal distension and failure to thrive appears between 6 months and 2 years of age affecting their weight and growth. In some it remains undiagnosed until adulthood., ${ }^{2,15}$

Celiac disease with atypical symptoms is characterized by few or no gastrointestinal manifestations and its recognition is partly responsible for the increased prevalence. Silent $\mathrm{CD}$ is associated with asymptomatic individuals but have a positive serologic test and typical histopathological changes. These patients are usually detected via screening of high risk individuals. Latent CD is defined by a positive serologic result but lack of symptoms and villous atrophy on biopsy but later may developed symptoms and histopathological changes. ${ }^{10}$

Prompt diagnosis and treatment of CD not only eases symptoms and improves quality of life but also has the potential to decrease long term risks for lymphoma, gastrointestinal carcinoma, dermatitis herpetiformis, osteopathy, endocrine abnormalities and other autoimmune disorders. $^{2}$ The overall risk of cancer is almost twice in patients with CD compared to the general population. Adherence to a gluten free diet is thought to reduce the risk of lymphoma. ${ }^{10}$

\section{DIAGNOSIS}

Guidelines on CD diagnosis have been published by gastrointestinal organizations since 2012. These guidelines include the combined use of biopsy and serologic analyses for diagnosis. According to American College of Gastroenterology (ACG) 2013 CD guidelines combination of both small intestinal biopsy and serologic tests (anti tissue Transglutaminase (tTG) or anti- deamidated gliadin peptide (DGP) are recommended for diagnosis of CD. ${ }^{15}$ Guideline by European Society of Pediatric Gastroenterology, Hepatology and Nutrition (ESPGHAN) in 2012 proposed a non invasive method of diagnosing $\mathrm{CD}$ in pediatric patients. These patients with symptoms consistent with $C D$ can be diagnosed without biopsy confirmation if they have an IgA tTG titre $>10$ fold above the upper limit of normal, a positive endomysial antibody (EMA) in a separate blood sample and carry the HLA DQ2 or DQ8 haplotype. ${ }^{16}$ The British Society of Gastroenterology recommendations for adult CD suggest that serologic tests either tTG, EMA or DGP should be done followed by small intestinal biopsy for a definitive diagnosis. ${ }^{1}$ Recent guidelines from the World Gastroenterologhical Association recommend serologic tests including anti- tTG and / or anti- EMA or anti- DGP for diagnosis and biopsy suggested but not considered mandatory for CD diagnosis. ${ }^{1,17}$

The gold standard of diagnosis is the small bowel mucosal biopsy together with positive serology. In 1992, Marsh reviewed the intensity of mucosal damage observed in treated CD patients who were confronted with increased amounts of gluten. A modified Marsh classification is now widely used in diagnosing CD in clinical practice. ${ }^{17,18}$

\section{SMALL INTESTINAL BIOPSY}

Histologic damage is considered characteristics but not pathognomonic of CD as similar lesions are seen in several other disorders. CD affects the mucosa of the proximal small intestine and less to the distal small intestine. The severity and extent of the histological damage appear to correlate with the intensity of the clinical symptoms. At least four biopsy samples must be taken, three from the second part of the duodenum distal to the papilla and one from the duodenal bulb. A second biopsy can be done in selected patients who have positive autoantibodies such as EMA. ${ }^{17}$

The characteristic histopathological findings seen are:

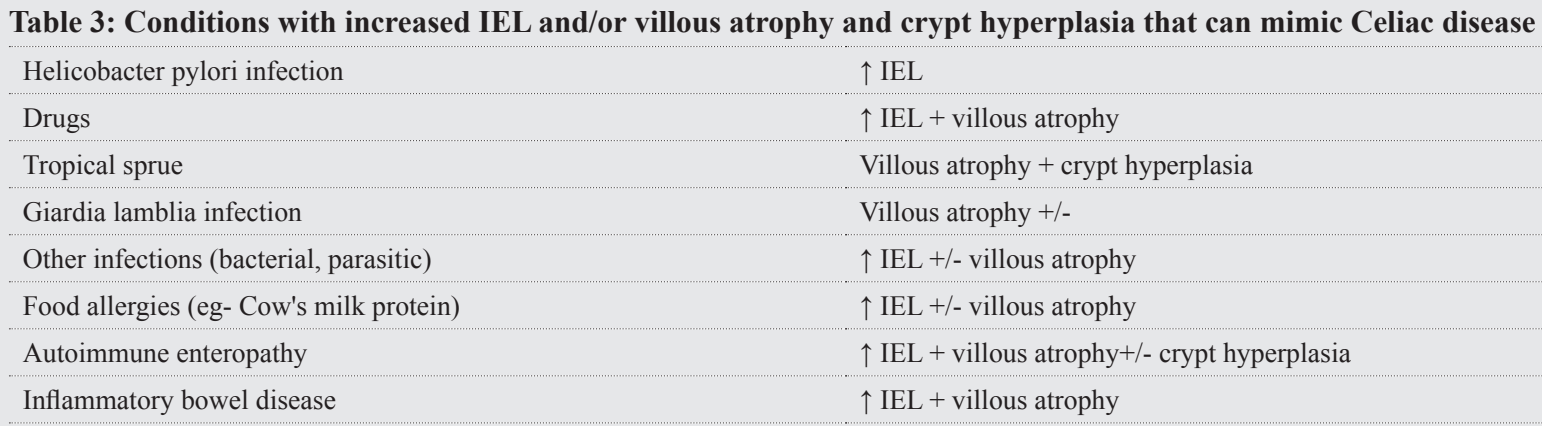




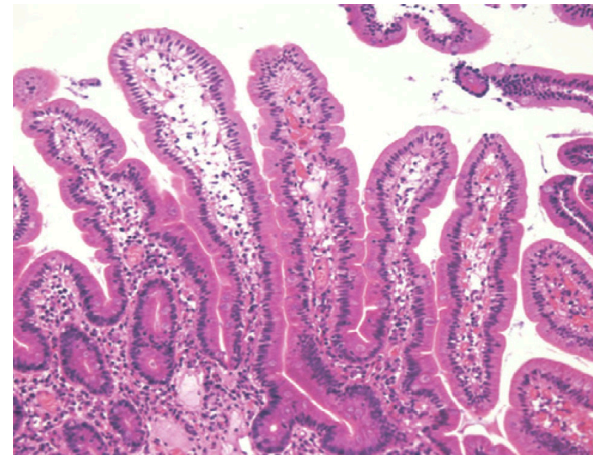

Figure 1: Normal duodenal mucosa (villous crypt ratio: $3: 1$ and IELs within normal range; HE stain $\times 100)$.

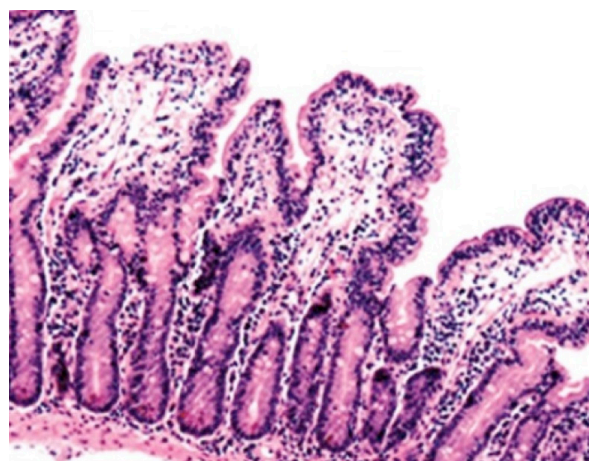

Figure 3: Partial villous atrophy and diffuse increase in IELs(HE stain x100).

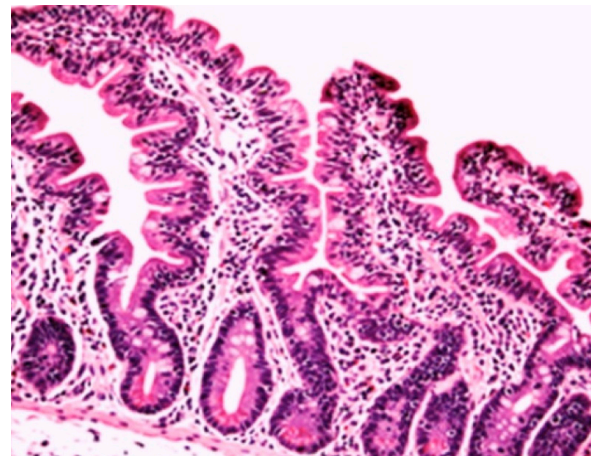

Figure 2: Increased numbers of IELS (HE stain $\times 100)$.

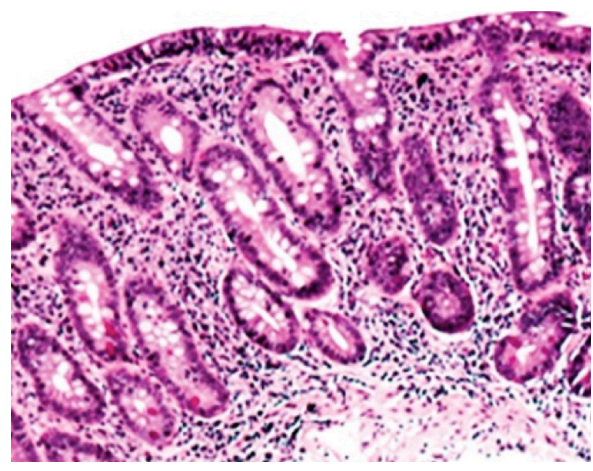

Figure 4: Total villous atrophy and diffuse increase in IELs (HE stain x100). blunted or atrophic villi, crypt hyperplasia, mono nuclear cell infiltration in the lamina propria, epithelial changes, including structural abnormalities in epithelial cells and intraepithelial lymphocyte (IEL) infiltration.10,17 Increased in IEL is the first and most sensitive marker of the effects of gluten on the small bowel mucosa and is therefore the major histological feature of Celiac disease. It is suggested that a clustering of lymphocytes $(>/=12)$ in the epithelium at the tips of villi and extending evenly down along the sides of the villus are a clue that $\mathrm{CD}$ may be present. Increased cellularity in the lamina propria is another important histological finding of Celiac disease. Plasma cells, lymphocytes and eosinophils are increased in number, particularly in the upper half of the mucosa. Enterocyte damage resulting in vacuolated cytoplasm is seen in severe injury. Other features of sever injury like villous atrophy and crypt hyperplasia can only be assessed in well oriented sections. Villous crypt ratios can be assessed in four or more crypts in parallel. Villi overlying and adjacent to lymphoid nodules/ follicles are normally blunted or absent and such areas should not be chosen for analysis. 9

The modified Marsh classification of gluten induced small intestinal damage helps to interpret the histopathological findings ranging from normal mucosa to completely flat villi. (Table- 1)
A correct histopathological diagnosis requires factor related to number of samples, sample quality, processing and reading. There are factors to be considered for ensuring reliable histological diagnosis. (Table 2)

However there are many other conditions with increased IEL and/or villous atrophy and crypt hyperplasia that can mimic Celiac disease.18 (Table 3)

A number of serological markers have been shown repeatedly in many studies to be highly sensitive and specific for untreated Celiac disease. There are two groups of serological tests, autoantibodies- EMA, tTG antibody and antibodies targeting the offending agent (AGAs) which is now considered obsolete for diagnostic purposes because of their lower sensitivity and specificity and antibodies against synthetic deamidated gliadin peptides (DGPs). These antibodies are based on immunoglobulin A (IgA) or immunoglobulin $\mathrm{G}$ (IgG). IgG based tests are useful for detecting $\mathrm{CD}$ in IgA deficient patients. 17

IgA EMA test is moderately sensitive (80\%) and highly specific (almost 100\%) for untreated (active) Celiac disease. Anti tTG antibodies are highly sensitive and specific for the diagnosis of Celiac disease. Deamidated gliadin peptides antibodies were introduced a few years ago and recently two DGP tests are combined in a single assay including IgA 
and $\operatorname{IgG}$ tTG determination. ${ }^{17}$ Sensitivity and specificity differs from patient to patient in Celiac disease. Therefore choosing the most appropriate serologic test in different clinical scenarios is a wise thing. For confirmation of gluten dependence in patients with enteropathy, IgA EMA, IgA tTG and IgG and IgA DGP gives the good result. IgG DGP is helpful in IgA deficient patients and for some EMAnegative and tTG negative patients. ${ }^{17}$ Serologic testing is very useful for screening patients with suspected $C D$ as early detection is essential to prevent the complications of Celiac disease. ${ }^{19}$

After the diagnosis, patients should be advised for the importance of strict adherence to the diet. Serological screening of first degree and second degree relatives should be considered. Persistence of symptoms is almost always caused by continued ingestion of gluten. ${ }^{17}$

Refractory CD is diagnosed when symptoms persist and when there is villous atrophy and failure to respond to a gluten free diet. This may be primary (occurring at the time of presentation) or secondary (after an initial response to a gluten free diet). It is usually diagnosed after the age of $50 .{ }^{17}$ Possible causes of refractory $\mathrm{CD}$ are unrecognized intake of gluten, lack of adherence to a gluten free diet and development of lymphoma. Possible findings in histology is the thickened subepithelial collagen layer, mucosal thinning and subcryptal mononuclear inflammation and evidence of lymphoma. ${ }^{9}$ There are two subtypes of refractory CD, type I with normal IEL and type II with clonal expansion of IEL and an aberrant phenotype lacking CD3, CD8 and T cell receptors. Type II refractory CD is the most sever form and is considered to be a form of low grade intraepithelial lymphoma. ${ }^{17}$

When screening small bowel biopsy several features have to be considered before giving the diagnosis. ${ }^{20}$ Hence, checklist based, templated pathology report can be beneficial to ensure capturing and reporting all relevant histopathological features. ${ }^{10}$

\section{Reports should include:}

- Site and number of biopsy specimens with a comment on specimen orientation.

- Villous to crypt ratio: normal (3:1 to 5:1) or abnormal

- Presence and degree of villous atrophy: normal or atrophicmild (partial), Moderate (subtotal) or severe (total)

- Increase in IEL counts with use of immunohistochemistry for $\mathrm{CD} 3$ in equivocal cases.

o Normal: Fewer than 25IELs /100 enterocytes

o Borderline increased: 25-29 IELs/100 enterocytes o Definitely increased: at least 30 IELs /100 enterocytes

- Presence / absence of surface epithelium damage

- Presence / absence of subepithelial collagen

- Lamina propria inflammation: type and degree

- Other: Clinical information and serology results, descriptive diagnosis including differential diagnosis and histopathological impression consistent with or suggestive of Celiac disease.

\section{CONCLUSION}

Celiac disease is a chronic systemic immune mediated disorder associated with variable small intestinal mucosal injury triggered by gluten in genetically predisposed individuals. Small bowel biopsy remains an essential component to the screening and diagnosis of celiac disease. With improved sensitivity and specificity of serologic testing and growing awareness among the clinicians, the diagnosis of $\mathrm{CD}$ is becoming easier and accurate. However, because of varied clinicopathological spectrum of the disease and many entities in the differential diagnosis of gluten sensitive enteropathy, diagnosis depends on good clinicopathological communication. The pathology report should be brief, descriptive and summary of the salient histopathological findings which can be easily assimilated by clinician. Serological testing or re biopsy can be recommended if indicated to promote standardisation and consensus among pathologists and clinicians.

\section{REFERENCES}

1. Kaswala DH, Veeraraghavan G, Kelly CP, Leffler DA. Celiac disease: diagnostic standards and dilemmas. Diseases. 2015;3:86101. Crossref

2. Ensari A. Gluten sensitive enteropathy (celiac disease) - controversies in diagnosis and classification. Arch Pathol Lab Med. 2010;134:82635. Crossref

3. Dickson BC, Streutker CJ, Chetty R. Celiac disease: an update for pathologist. J Clin Pathol. 2006;59:1008-16. Crossref

4. Osman MT, Al- Nasiry SA, Fayadh MH, Taha BI. Clinicopathological study of Iraqi patients group suspected to have celiac disease. Innov J Med Health Sci. 2012;2:98-103.

5. Elli L, Zini E, Tomba C et al. Histological evaluation of duodenal biopsies from celiac patients: the need for different grading criteria during follow up. BMC Gastroenterol.2015;15:133-40. Crossref

6. Villanacci V, Ceppa P, Tavani E, Vindigni C, Volta U. Celiac disease: the histology report. Dig Liver Dis. 2011;43: 385-95. Crossref

7. Sollid LM, Jabri B. Is celiac disease an auto immune disorder? Curr Opin Immunol. 2005;17:595-600. Crossref

8. Green PH, Jabri B. Celac disease. Annu Rev Med. 2006;57:207-21. Crossref

9. Antoniolo DA. Celiac disease: a progress report. Mod Pathol. 2003;16:342-6. Crossref

10. Bao F, Green PHR, Bhagat G. An update on celiac disease histopathology and the road ahead. Arch Pathol Lab med. 2012;136:735-45. Crossref 
11. Tapia AR, Murray JA. Celiac disease. Curr Opin Gastroenterol. 2010;26: 116-22. Crossref

12. Shahraki T, Hill ID. Prevalence of celiac disease in first degree relative of children in Sistan and Baluchestan province (Iran). J Dig Dis. 2016;10:1751-2980. Crossref

13. Isaac DM, Wu J, Mager DR, Turne JM. Managing the pediatric patient with celiac disease: a multidisciplinary approach. J Multidiscip Healthc. 2016;13:529-36. Crossref

14. Capriati T, Francavilla R, Castellaneta S, Ferretti, Diamanti A. Impact of the birth's season on the development of celiac disease in Italy. Eur J Pediatr. 2015;174:1657-63. Crossref

15. Rubio-Tapia A, Hill ID, Kelly CP, Calderwood AH, Murray JA. ACG clinical guidelines: Diagnosis and management of celiac disease. Am J Gastroenterol. 2013;108:656-76. Crossref
16. Poddar U. Celiac disease: clinical features and diagnostic criteria. Indian J Pediatr. 1999;66: 21-5.

17. Bai JC, Fried M, Roberto $\mathrm{G}$ et al. Celiac disease. World Gastroenterology Organisation global guidelines. 2012.

18. Svajdler M, Daum O, Rychly B. Diagnosing celiac disease: role of the pathologists. Intl J Celiac Dis. 2014;2:70-5. Crossref

19. Rashid M, Lee J. Serologic testing in celiac disease: practical guide for clinicians. Can Fam Physician. 2016;62:38-43. Crossref

20. Bao F. Bhagat G. Histopathology of celiac disease. Gastrointest Endosc Clin N Am. 2012;22:679-94. Crossref 